\title{
Interface Interactions Modulating Desensitization of the Kainate-Selective Ionotropic Glutamate Receptor Subunit GluR6
}

\author{
Yihong Zhang, ${ }^{1}$ Naushaba Nayeem, ${ }^{1}$ Max H. Nanao, ${ }^{2}$ and Tim Green ${ }^{1}$ \\ ${ }^{1}$ Department of Pharmacology, School of Biomedical Sciences, University of Liverpool, Liverpool L69 3GE, United Kingdom, and ${ }^{2}$ Exelixis Inc., South San \\ Francisco, California 94083
}

\begin{abstract}
Ionotropic glutamate receptors from the AMPA and kainate subfamilies share many functional and structural features, but it is unclear whether this similarity extends to the molecular mechanisms underlying receptor desensitization. The current model for desensitization in AMPA receptors involves the rearrangement of dimers formed between subunit agonist binding domains. Key evidence for this has come from a single point mutant (from leucine to tyrosine) that abolished desensitization and that was shown to stabilize the binding domain dimer. However, the desensitization of kainate receptors appears to differ from that of AMPA receptors in several key respects. Although the kinetics of AMPA receptor gating and desensitization are consistent with channels formed from two dimers, similar evidence for the functional involvement of dimers has not been found in kainate receptors. Furthermore, despite the homolog of the nondesensitizing tyrosine in AMPA subunits also being a tyrosine in wild-type kainate subunits, these receptors desensitize rapidly and completely. Using mutagenesis based on the crystal structure of the glutamate receptor subunit GluR6 S1S2 domain in complex with domoate, we identified four residues neighboring this tyrosine that differ between AMPA and kainate subunits and that contribute to the different desensitization kinetics of these receptors. Detailed analysis of the effects of mutations at these sites confirms that there is in fact a common general mechanism for desensitization in non-NMDA receptors, dependent on the stability of the binding domain dimer interface, and reveals the existence of potential agonist-specific desensitization pathways.
\end{abstract}

Key words: glutamate; kainate receptor; desensitization; S1S2 domain; dimer interface; mutagenesis

\section{Introduction}

The kinetics of ionotropic glutamate receptor (iGluR) desensitization affect the frequency and amplitude of excitatory responses in the mammalian CNS (Jones and Westbrook, 1996). Compounds that enhance AMPA receptor signaling by reducing desensitization are being studied as potential cognitive enhancers (Lynch, 2002). It is therefore important to understand the molecular basis of iGluR desensitization. Significant progress has been made in this, particularly for AMPA receptors. It is now thought that AMPA receptor desensitization requires the rearrangement of a twofold dimer formed between subunit agonist binding (S1S2) domains in the receptor complex (Sun et al., 2002). This model was based partly on the discovery that a single point mutation in GluR3 (L507Y) blocked desensitization (Stern-Bach et al., 1998). This site is within a dimer interface

Received April 28, 2006; revised Aug. 21, 2006; accepted Aug. 22, 2006.

This work was supported by the United Kingdom Medical Research Council. We thank Steve Heinemann (Salk Institute, La Jolla, CA) for the GluR6 cDNA and David Wyllie (University of Edinburgh, Edinburgh, UK) for advice and assistance on the use of rapid perfusion systems. We are also grateful to Geoff Swanson (Northwestern University, Evanston, IL) for initial recordings of the E787 site mutants and John Quayle (University of Liverpool, Liverpool, UK) for critical reading of this manuscript.

Correspondence should be addressed to Tim Green, Department of Pharmacology, School of Biomedical Sciences, University of Liverpool, Ashton Street, Liverpool L693GE, UK. E-mail: tpgreen@liv.ac.uk.

DOI:10.1523/JNEUROSCI.2750-06.2006

Copyright $\odot 2006$ Society for Neuroscience ～0270-6474/06/2610033-10\$15.00/0 observed in x-ray structures (Armstrong and Gouaux, 2000), and dimer stability has been shown to be inversely related to the degree of desensitization (Sun et al., 2002). The desensitization block of the leucine to tyrosine mutation (L483Y in GluR2) results from the formation of a stabilizing cation- $\pi$ interaction with a lysine in the adjacent subunit (Sun et al., 2002). The "dimerrearrangement" desensitization model is supported by a number of observations. Allosteric potentiators (Jin et al., 2005) bind at the dimer interface, whereas disruptive mutations within the interface accelerate the rate of desensitization (Horning and Mayer, 2004). Kinetic (Bowie and Lange, 2002; Yelshansky et al., 2004) and electron microscopic (Tichelaar et al., 2004) studies also show that the key functional unit of AMPA receptors is a dimer. Most recently, it has been shown that NMDA subunit S1S2 domains form twofold dimers that are stabilized by mutations equivalent to R2 L483Y (Furukawa et al., 2005).

The picture is much less clear for kainate (KA) receptors. Although a twofold symmetric dimer is evident in the structure of GluR6 S1S2 in complex with domoate (Nanao et al., 2005), other evidence suggests that AMPA and kainate receptors desensitize via different mechanisms. At several sites within the dimer interface in which mutations to GluR2 had promoted desensitization (Horning and Mayer, 2004), equivalent changes in GluR6 unexpectedly slowed desensitization (Fleck et al., 2003). Furthermore, the dimeric associations identified by kinetic studies in AMPA 
subunits were either not observed or were weaker in kainate subunits (Bowie and Lange, 2002; Yelshansky et al., 2004). Gating mechanisms also differ, with AMPA subunits gating independently (Rosenmund et al., 1998; Smith et al., 2000) and kainate subunits gating in a concerted manner (Smith and Howe, 2000). It is therefore interesting that, although kainate subunits contain a tyrosine at the site equivalent to R2 L483 (R6 Y521), they desensitize on a millisecond timescale. To investigate why this is and whether dimer rearrangement plays a role in kainate receptor desensitization, we looked for differences in the dimer interfaces of GluR6 and GluR2 (see Fig. 1) (Armstrong and Gouaux, 2000; Nanao et al., 2005). Using directed mutagenesis and functional characterization, we studied the effects on GluR6 desensitization of mutations to sites surrounding Y521.

\section{Materials and Methods}

Mutagenesis and structure analysis. All mutagenesis was performed on a rat GluR6 cDNA clone carrying a glutamine codon at the Q/R site. Residue numbering of GluR6 is for the full-length subunit and of GluR2 is for the mature polypeptide, consistent with conventional usage in the literature (Fig. 1). Mutants were generated using the Quikchange protocol (Stratagene, La Jolla, CA). In brief, a pair of complementary oligonucleotide primers containing the mutation (synthesized by SigmaGenosys, Haverhill, UK), were used in a PCR reaction $\left(95^{\circ} \mathrm{C}\right.$ for $1 \mathrm{~min}$, $52-61^{\circ} \mathrm{C}$ for $1 \mathrm{~min}, 68^{\circ} \mathrm{C}$ for $16 \mathrm{~min}$ for 18 cycles) with $2.5 \mathrm{U}$ of $P f u$ turbo polymerase (Stratagene). A DpnI digest of the PCR mix was then performed for $1 \mathrm{~h}$ at $37^{\circ} \mathrm{C}$. Competent DH5 $\alpha$ Escherichia coli (Invitrogen, Paisley, UK) were transformed using standard procedures. All mutants were confirmed by sequencing of the entire open reading frame.

Investigation of potential residue interactions was performed using the structure determined for the GluR6 S1S2 domoate complex [Protein Data Bank accession number 1YAE (Nanao et al., 2005)]. Distance measurements were for interactions at the interface between protomers $B$ and D. Figures of the structure were generated using MACPYMOL (http://pymol.sourceforge.net).

Cell culture. HEK 293 cells (European Collection of Cell Cultures, Porton Down, UK) were maintained at $37^{\circ} \mathrm{C}, 5 \% \mathrm{CO}_{2}$ in DMEM (Invitrogen), supplemented with $10 \%$ fetal calf serum and penicillin/streptomycin. Cells were transiently transfected with cDNA plasmids encoding GluR6 and mutants thereof, using either calcium phosphate (for surface labeling and binding assays) or Lipofectamine 2000 (for electrophysiology) (Invitrogen).

Cell surface biotinylation. After transfection (48-54 h), HEK 293 cells were harvested, washed with PBS, and biotinylated using $6 \mathrm{mg} / \mathrm{ml}$ sulfoNHS-SS-biotin (Pierce via Perbio Science UK, Cramlington, UK) in PBS at $4^{\circ} \mathrm{C}$ for $1 \mathrm{~h}$. Unreacted biotinylation reagent was quenched with $\mathrm{NH}_{4} \mathrm{Cl}(15 \mathrm{~mm})$, and the cells were washed in PBS and lysed in radioimmunoprecipitation assay (RIPA) buffer ( $1 \%$ Triton X-100, $1 \%$ sodium deoxycholate, $0.1 \%$ SDS, $150 \mathrm{~mm} \mathrm{NaCl}, 2 \mathrm{~mm}$ EDTA, and $50 \mathrm{~mm}$ Tris$\mathrm{HCl}, \mathrm{pH}$ 7.4) containing a protease inhibitor cocktail (Sigma-Aldrich, Poole, UK). After $2 \mathrm{~h}$ on ice, samples were centrifuged $(100,000 \times g, 30$ $\min , 4^{\circ} \mathrm{C}$ ), and the supernatants were incubated with immobilized Neutr-Avidin (Pierce) overnight at $4^{\circ} \mathrm{C}$. After RIPA buffer washes, biotin-labeled proteins were recovered by the addition of SDS-PAGE loading buffer. Estimations of protein concentrations were made using BCA reagents (Pierce). Equal amounts of total and surface proteins in each sample were separated by SDS-PAGE and immunoblotted using a GluR6/7 polyclonal antibody (Upstate, Milton Keynes, UK). Blots were incubated with an HRP-conjugated anti-rabbit secondary (Vector Laboratories, Peterborough, UK) and developed using SIGMAFast DAB peroxidase substrate (Sigma-Aldrich).

Electrophysiology. HEK 293 cells were cotransfected with GluR6 and a plasmid expressing CD8. Whole-cell recordings were performed $12-72 \mathrm{~h}$ after transfection at room temperature with an EPC 10 amplifier (HEKA Elektronik via Digitimer, Welwyn Garden City, UK). The holding potential was set to $-70 \mathrm{mV}$. Patch pipettes $(2-5 \mathrm{M} \Omega$ ) were filled with a solution containing the following (in mM): $110 \mathrm{CsF}, 30 \mathrm{CsCl}, 4 \mathrm{NaCl}, 0.5$

A

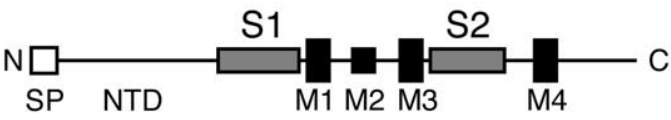

B

R6 S1 R2 S1

\section{R6 S1} R2 S1

R6 S1

R2 S1

R6 S1 R2 S1

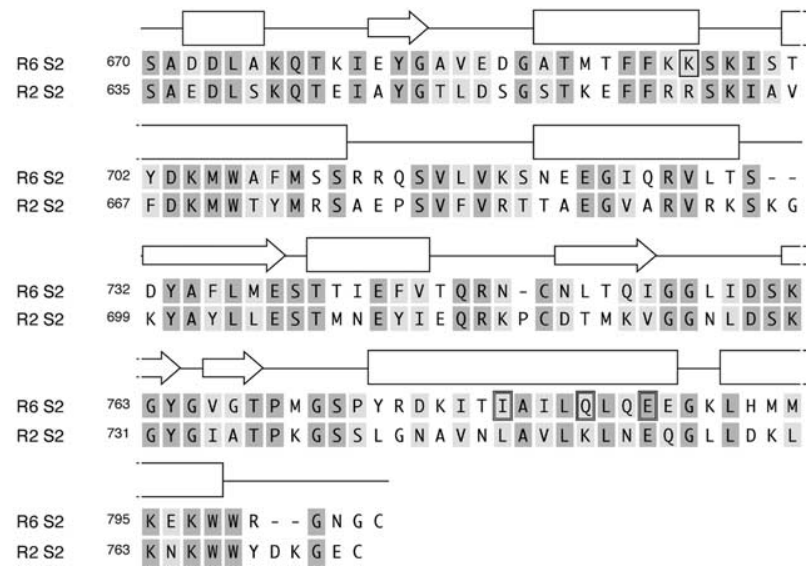

Figure 1. Location of mutated residues within the GluR6 S1S2 domain. $\boldsymbol{A}$, Domain organization in GluR6. The locations of the $S 1$ and $S 2$ domains are shown relative to the membrane domains (M1-M4), N-terminal domain (NTD), and signal peptide (SP). B, Alignment of GluR6 (R6) and GluR2 (R2) S1 and S2 domains, with sequence similarity indicated by shading (dark gray for identities, light gray for conserved). Residue numbering as used in text is shown on the left. Secondary structure elements in GluR6 are indicated by rectangles ( $\beta$-strands) and arrows ( $\alpha$-helices). Residues mutated in this study are boxed, with single and double lines used to indicate on which side of the interface they lie relative to Y521 (intra and inter, respectively).

$\mathrm{CaCl}_{2}, 10 \mathrm{HEPES}$, and 5 EGTA, adjusted to $\mathrm{pH} 7.3$ with $\mathrm{CsOH}$. The external bath solution contained the following (in $\mathrm{mM}$ ): $150 \mathrm{NaCl}, 2.8$ $\mathrm{KCl}, 1.8 \mathrm{CaCl}_{2}, 1.0 \mathrm{MgCl}_{2}$, and 10 HEPES, adjusted to $\mathrm{pH} 7.3$ with $\mathrm{NaOH}$. Kainic acid was purchased from Tocris Cookson (Avonmouth, UK).

Rapid agonist application was achieved by using a Burleigh LSS-3200 piezo-based perfusion system (Scientifica, Harpenden, UK). Control and agonist solutions were driven simultaneously through the two parallel barrels of a theta tube (Hilgenberg, Malsfeld, Germany), pulled to an outside diameter of $\sim 300 \mu \mathrm{m}$. The rate of solution exchange was $<250$ $\mu$ s as determined by open-tip junction currents. Recordings were performed on small-diameter cells $(\sim 20 \mu \mathrm{m})$, lifted into the perfusion stream. For control responses [GluR6 wild type (WT)], the $20-80 \%$ rise time was $<1 \mathrm{~ms}$. Data were acquired by Pulse 8.65 (HEKA Elektronik), stored directly to a computer, and analyzed off-line using Pulsefit 8.65 or AxonGraph 4.9. Exponential decays were fitted using the "Simplex" least-squares algorithm, typically from $90-100 \%$ of peak to the steadystate level. When responses showed slow initial desensitization, desensitization rates were determined starting from $75-90 \%$ of peak response. In all cases, this resulted in a single-exponential fit. For mutants with long desensitization time constants $\left(\tau_{\mathrm{des}}\right)$, application times of up to $7 \mathrm{~s}$ were 
Table 1. Functional analysis of mutants made to region surrounding Y521 in GluR6

\begin{tabular}{|c|c|c|c|c|c|c|c|c|}
\hline \multirow[b]{2}{*}{ Mutant } & \multicolumn{4}{|l|}{ Glutamate } & \multicolumn{4}{|l|}{ Kainate } \\
\hline & $I_{\text {peak }}(\mathrm{nA})$ & $\tau_{\text {des }}(\mathrm{ms})$ & \%des & $n$ & $I_{\text {peak }}(\mathrm{nA})$ & $\tau_{\text {des }}(\mathrm{ms})$ & \%des & $n$ \\
\hline Wild type & $6.4 \pm 0.3$ & $5.7 \pm 0.1$ & $99.7 \pm 0.04$ & 80 & $4.6 \pm 0.3$ & $4.6 \pm 0.1$ & $99.5 \pm 0.1$ & 47 \\
\hline K525E & $3.0 \pm 0.4$ & $6.3 \pm 0.4$ & 100 & 10 & $1.1 \pm 0.2$ & $3.7 \pm 0.5$ & $99.9 \pm 0.1$ & 8 \\
\hline $\mathrm{I} 780 \mathrm{~A}$ & $0.2 \pm 0.1$ & $1.0 \pm 0.1$ & 100 & 9 & $0.03 \pm 0.01$ & $0.9 \pm 0.2$ & 100 & 4 \\
\hline I780F & $1.4 \pm 0.2$ & $1.3 \pm 0.1$ & 100 & 6 & $0.2 \pm 0.04$ & $1.3 \pm 0.2$ & 100 & 6 \\
\hline I780L & $9.7 \pm 0.6$ & $17.8 \pm 1.4$ & $98.1 \pm 0.6$ & 12 & $8.0 \pm 0.7$ & $42.7 \pm 3.0$ & $87.1 \pm 1.7$ & 10 \\
\hline I780M & $6.1 \pm 0.5$ & $4.3 \pm 0.2$ & $99.9 \pm 0.1$ & 12 & $4.5 \pm 0.5$ & $4.3 \pm 0.3$ & $99.5 \pm 0.3$ & 11 \\
\hline I780S & $2.3 \pm 0.5$ & $1.2 \pm 0.1$ & 100 & 9 & $0.5 \pm 0.1$ & $1.2 \pm 0.1$ & 100 & 9 \\
\hline I780V & $3.7 \pm 0.6$ & $2.6 \pm 0.1$ & 100 & 10 & $2.2 \pm 0.5$ & $2.5 \pm 0.3$ & 100 & 9 \\
\hline Q784K & $7.4 \pm 0.7$ & $7.6 \pm 0.6$ & $99.7 \pm 0.2$ & 12 & $6.2 \pm 0.5$ & $5.3 \pm 0.6$ & $99.6 \pm 0.2$ & 10 \\
\hline K525E & $4.7 \pm 0.9$ & $15.1 \pm 0.1$ & $99.9 \pm 0.03$ & 12 & $3.7 \pm 0.6$ & $5.7 \pm 0.3$ & $99.7 \pm 0.3$ & 11 \\
\hline \multicolumn{9}{|l|}{ Q784K } \\
\hline Y521L & $7.4 \pm 0.9$ & $6.5 \pm 0.4$ & $99.9 \pm 0.02$ & 11 & $5.5 \pm 0.8$ & $4.9 \pm 0.4$ & $99.4 \pm 0.2$ & 10 \\
\hline \multicolumn{9}{|l|}{ K525E } \\
\hline \multicolumn{9}{|l|}{ Q784K } \\
\hline K525E & $7.2 \pm 0.8$ & $61.5 \pm 4.1$ & $98.2 \pm 0.7$ & 10 & $6.1 \pm 0.9$ & $132 \pm 12$ & $86.9 \pm 2.8$ & 10 \\
\hline \multicolumn{9}{|l|}{ I780L } \\
\hline Q784K & & & & & & & & \\
\hline
\end{tabular}

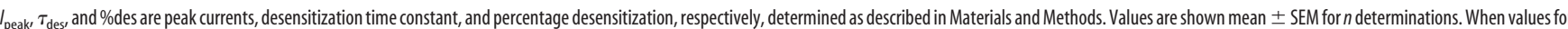

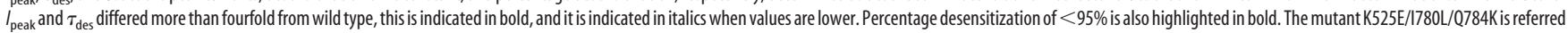
to as the triple mutant in the text.

used to ensure currents either reached steady state (for determining desensitization rates) or provided stable steady-state responses (for determining the extent of desensitization). Steady-state current values were used to estimate $\Delta G_{\text {des }}$ (in kilocalories per mole) for WT and mutant GluR6, calculated from the extent of desensitization as described by Sun et al. (2002), and using a temperature of $294 \mathrm{~K}$.

Membrane preparation and radioligand binding assays. Membrane preparations and $\left[{ }^{3} \mathrm{H}\right]$ kainate saturation and displacement assays were performed as described previously (Swanson et al., 1997), using $0.1 \%$ Triton $\mathrm{X}-100$ as a surfactant in the binding assays. [Vinylidene${ }^{3} \mathrm{H}$ ] kainic acid (specific activity, $47-58 \mathrm{Ci} / \mathrm{mmol}$ ) was purchased from PerkinElmer Life and Analytical Sciences (Beaconsfield, UK). Saturation binding was performed using $\left[{ }^{3} \mathrm{H}\right]$ kainate concentrations between 1 and $50 \mathrm{~nm}$. Nonspecific binding was defined in the presence of $1 \mathrm{~mm}$ glutamate.

\section{Results}

We tested the effects of mutations on receptor desensitization kinetics using whole-cell recordings in HEK 293 cells expressing WT or mutant GluR6. All mutants were tested with saturating concentrations of both Glu (3 mM) and KA (1 mM). Kainate is a partial agonist at GluR6, evoking currents $70-80 \%$ of those produced by glutamate (Table 1) (Swanson et al., 1997). In AMPA receptors, ligand efficacy is related to the degree of desensitization (Patneau et al., 1992; Jin et al., 2003), and it was therefore possible that KA might be a more sensitive probe for changes in receptor desensitization. In WT GluR6, both agonists evoked rapidly desensitizing currents of $6.4 \pm 0.3$ and $4.6 \pm 0.3 \mathrm{nA}$, respectively, with $\tau_{\text {des }}$ equal to $5.7 \pm 0.1$ and $4.6 \pm 0.1 \mathrm{~ms}$ (Table 1). Desensitization of GluR6 WT was also essentially complete for responses to both Glu and KA (99.7 \pm 0.04 and $99.5 \pm 0.1 \%$, respectively) (Table 1 ).

\section{GluR6 mutants K525E and Q784K slow desensitization kinetics in combination but not separately}

By comparing GluR2 and GluR6 S1S2 domain structures in which the twofold dimer was observed (Armstrong and Gouaux, 2000; Nanao et al., 2005), we identified differences that might account for the divergent desensitization behavior of these receptors (Nanao et al., 2005). Specifically, the intersubunit cation- $\pi$ interaction in the nondesensitizing R2 L483Y mutant (Sun et al., 2002) is absent from GluR6. In R2 L483Y, this interaction is formed between the tyrosine at position 483 and the lysine at position 752. GluR6 has a tyrosine at the position equivalent to L483 (Y521), but it lacks a positively charged residue at the site equivalent to K752 (R6 Q784). There may also be an intrasubunit cation- $\pi$ bond formed in GluR6 between the tyrosine and K525 (Fig. 2A), which in GluR2 is a glutamate (R2 E487). This interaction is not certain, however, because the electron density for K525 is weak (supplemental Fig. 1, available at www.jneurosci.org as supplemental material).

We therefore changed K525 and Q784 in GluR6 to the residues found in GluR2 [glutamate (E) and lysine (K), respectively] and tested the desensitization kinetics of these R6 K525E and Q784K single point mutants. For neither mutant was there any change in receptor kinetics in response to either Glu or KA compared with GluR6 WT (Table 1). The double mutant (R6 K525E/ Q784K), in contrast, exhibited 2.6-fold slower desensitization in response to Glu ( $\tau_{\text {des }}$ of $15.1 \pm 0.1 \mathrm{~ms}$ ) compared with WT (Fig. $2 B$, Table 1$)$. The desensitization kinetics in response to KA $\left(\tau_{\text {des }}\right.$ of $5.7 \pm 0.3 \mathrm{~ms}$ ) were also marginally slower than WT (Table 1). We confirmed that the effect of these changes on desensitization was attributable to the predicted interactions with residue Y521, by changing this site to leucine $(\mathrm{L})$ in combination with the double mutant. The resulting triple mutant (Y521L/K525E/Q787K) had desensitization kinetics close to WT (Fig. 2B, Table 1).

\section{Effects of additional mutations in the vicinity of GluR6 Y521}

The slower desensitization kinetics observed for the K525E/ Q784K double mutant were consistent with the formation of an intersubunit cation- $\pi$ interaction between the lysine replacing Q784 and the tyrosine replacing Y521. The effect, however, was clearly still a long way from the virtually complete block of desensitization observed for AMPA subunits containing aromatic residues at the R2 L483/R3 L507 site (Stern-Bach et al., 1998; Sun et al., 2002). We therefore sought to determine whether other residues surrounding this site might be contributing to the differences in desensitization between GluR6 WT and GluR2 L483Y. Revisiting the GluR6 S1S2 structure, we looked for neighbors of R6 Y521 that differ in GluR2/3 and GluR6. In the immediate vicinity of Y521, there is only one such residue, I780 (Fig. 2A). The homolog of this residue in GluR2 (L748) forms a hydropho- 
A
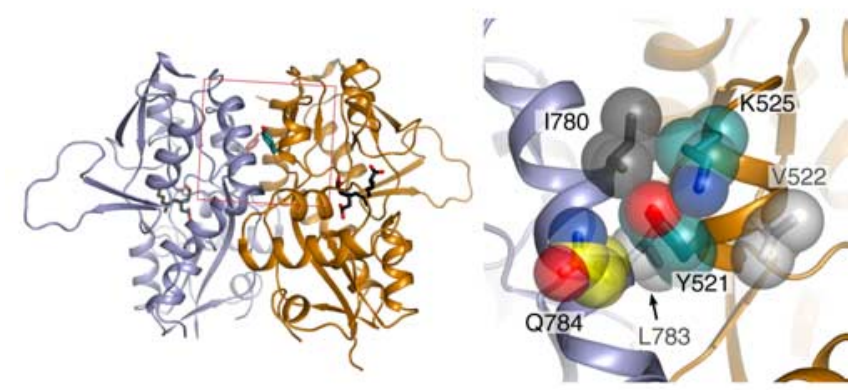

B
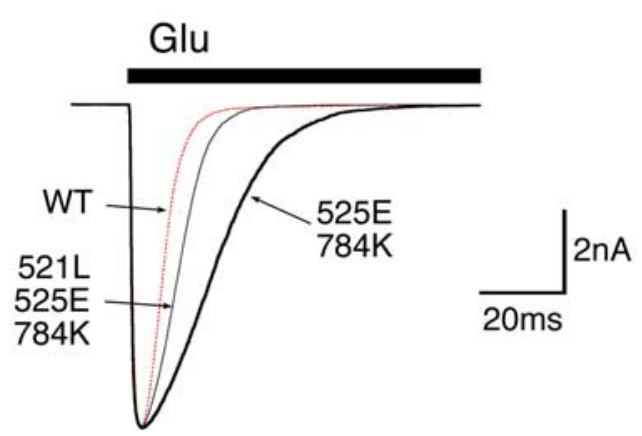

Figure 2. Desensitization of GluR6 is slowed by the formation of an intersubunit cation- $\pi$ interaction between residues 521 and 784. $\boldsymbol{A}$, View of GluR6 S1S2 dimer (Protein Data Bank accession number 1YAE) (Nanao et al., 2005). The left panel shows an overview of the dimer, with bound domoate in black and residue Y521 from both protomers in stick representation (protomer B in cyan; protomer $D$ in red). The dimer is orientated so that the linker replacing the M1-M3 membrane domains would be at the bottom. The right panel shows a close-up view of interface around residue Y521 (protomer B), showing the orientation of Y521 (blue) in relation to $\mathrm{K} 525$ (blue) on the same subunit and Q784 (yellow) on the adjacent subunit. Other neighboring residues (V522, 1780, and L783; gray) are shown. The red box on the left indicates the area shown on the right (the viewpoint is rotated clockwise and toward the viewer from the top). $\boldsymbol{B}$, Overlay of representative responses to $\mathrm{Glu}$ ( $3 \mathrm{~mm}$; black bar) recorded from HEK 293 cells expressing GluR6 WT (red dotted trace), R6 K525E/Q784K (thick trace), and R6 Y521L/K525E/ Q784K (thin trace). Response amplitudes were normalized to R6 K525E/Q784K.

bic pocket along with L751 (R6 L783) and the methylene groups of K752 (R6 Q784), into which the aromatic ring of R2 L483Y sits (Sun et al., 2002; Horning and Mayer, 2004). An additional hydrophobic residue, V484, is also located adjacent to L483 but again is conserved in GluR6 (as V522) (Fig. 2A). In GluR2, residue 748 has a role in dimer stability, because introduction of an L748A mutation into L483Y increases both the rate and extent of desensitization (Sun et al., 2002), and the L748A single mutant desensitizes 12-fold faster than wild type (Horning and Mayer, 2004).

If this site contributes to the desensitization differences between R6 WT and R2 L483Y, we would predict that R6 I780L would desensitize more slowly than GluR6 WT. This was indeed the case, with the rate of desensitization in response to Glu and KA for I780L slowed by 3.1-fold and 9.3-fold, respectively (Fig. $3 A, B$, Table 1). We hypothesized that the slower desensitization of I780L was the result of tighter packing with Y521 across the dimer interface, more closely mimicking the overall conformation of R2 L483Y. To test this, we mutated I780 to a number of different amino acids, varying the bulk and polarity. Of these mutants, mutation to methionine $(\mathrm{M})$ resulted in kinetics similar to WT in response to both Glu and KA (Fig. 3, Table 1). All of the other mutants tested [to alanine (A), phenylalanine $(\mathrm{F})$, serine $(\mathrm{S})$, and valine $(\mathrm{V})$ ] desensitized significantly faster than WT (Fig. 3 , Table 1). These data are also consistent with the hypothesis that
A

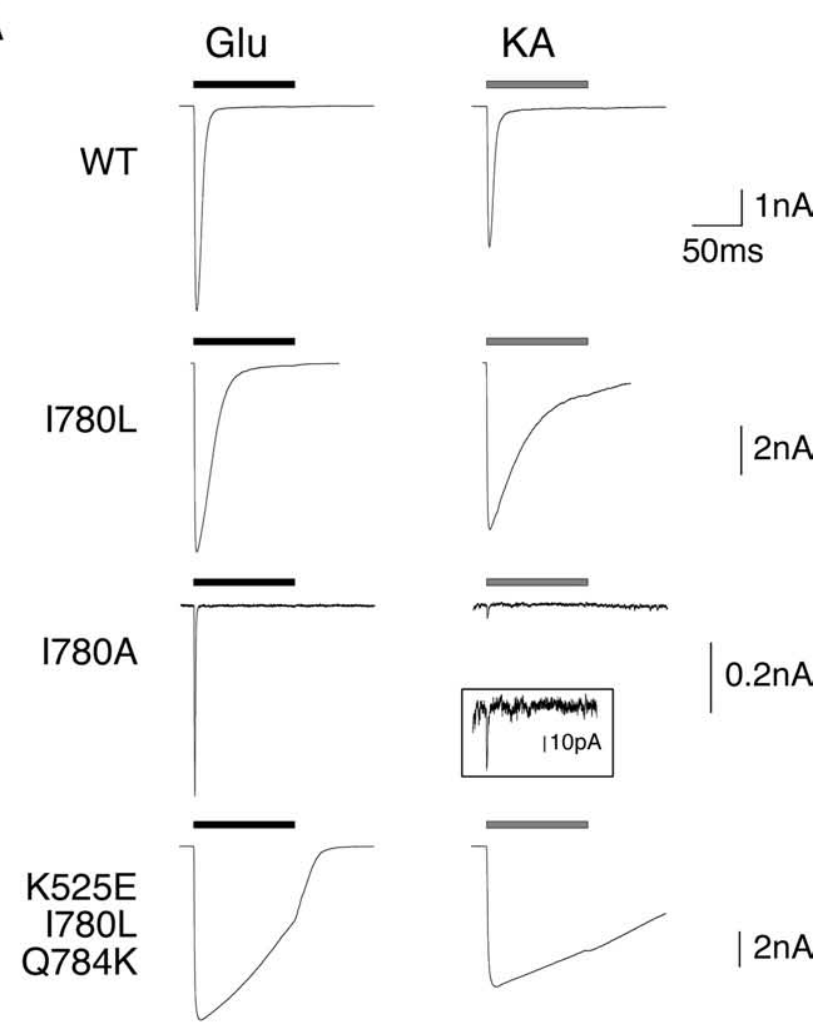

B

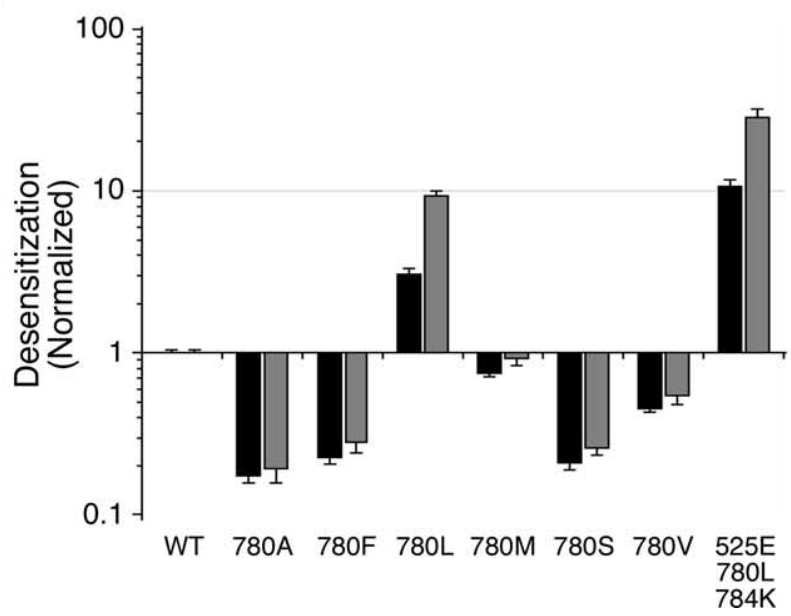

Figure 3. Role of residue 780 in GluR6 desensitization. $\boldsymbol{A}$, Representative responses to Glu (3 $\mathrm{mm}$; black bars) and KA (1 mm; gray bars), recorded from HEK 293 cells expressing GluR6 WT, R6 I780L, R6 I780A, and R6 K525E/I780L/Q784K. B, Bar graph showing $\tau_{\text {des }}$ for 1780 mutants and the triple mutant in response to applications of Glu (black) and KA (gray). Values (mean \pm SEM, normalized to WT) are plotted on a log scale. The number of determinations is given in Table 1.

the strength of the dimer interface around Y521 affects desensitization. From inspection of the structure, most of the mutations to I780 appear to worsen the fit between subunits, by either introducing hydrophobic voids (I780A, S, or V) or steric clashes (I780F). The minimal effect of the I780M mutation is possibly attributable to the torsional flexibility of methionine, allowing for a reasonable fit with Y521. For some of these mutants, currents were noticeably attenuated, particularly in response to KA. Kainate currents were much smaller for the I780S (11\% of WT), I780F (4\%), and I780A (0.7\%) mutants and reduced for I780V (48\%) (Fig. 3, Table 1). Currents in response to Glu were gener- 
A

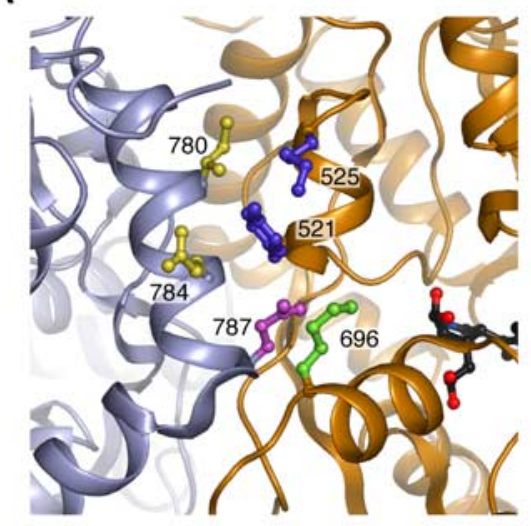

B
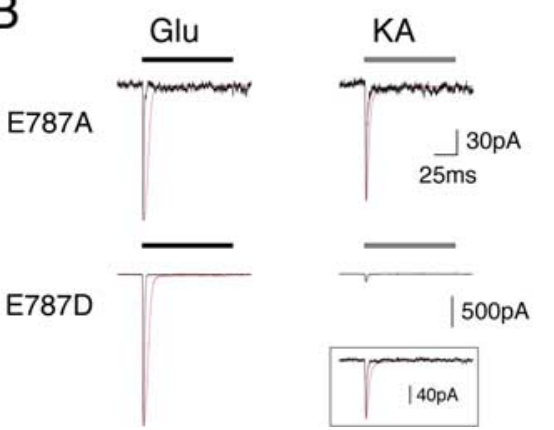

C
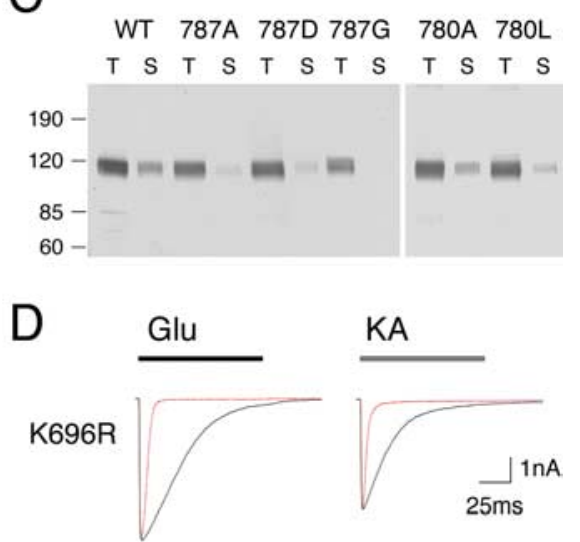

E

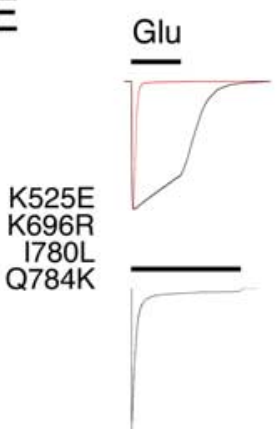

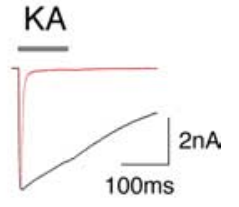

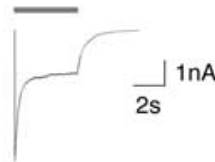

Figure 4. Two additional residues in the vicinity of residue Y521 with a large influence on desensitization kinetics. $\boldsymbol{A}$, GluR6 S1S2 dimer interface structure showing the location of $\mathrm{K} 696$ and E787 relative to Y521. Side chains are shown for Y521, E525 (both blue), and K696 (green) on one protomer and 1780, Q784 (both yellow), and E787 (pink) on the opposite protomer. Bound domoate is shown in black. $B$, Responses of R6 E787A and E787D to Glu ( $3 \mathrm{~mm}$; black bars) and KA (1 mm; gray bars). Normalized WT responses (red dotted traces) are overlaid, and an enlarged KA trace for E787D is shown inset. C, Immunoblot of surface-labeled HEK 293 cell lysates using anti-GluR6/7 polyclonal antisera. Biotinylation was performed on transiently transfected cells as described in Materials and Methods. Total $(\mathrm{T})$ and surface $(\mathrm{S})$ protein samples were loaded as indicated. Molecular mass (in kilodaltons) is shown to the left. $\boldsymbol{D}$, Responses of R6 K696R to Glu and KA as in $\boldsymbol{B}$ above. $\boldsymbol{E}$, Responses of quadruple mutant R6 K525E/ K696R//780L/Q784K to standard (100 ms; top traces) and extended ( $7 \mathrm{~s}$ for Glu or $4 \mathrm{~s}$ for KA; bottom traces) agonist applications.

ally less affected, with all of these mutants except I780A (in which currents were only $3 \%$ of WT) giving robust responses to glutamate, ranging from 22 to $58 \%$ of WT (Fig. 3, Table 1). Overall, for mutants with faster desensitization KA efficacy was reduced $\left(I_{\mathrm{KA}} /\right.$ $I_{\mathrm{Glu}}$ of $0.15-0.59$ compared with 0.72 for WT), whereas for the slowly desensitizing I780L mutant, KA efficacy was increased $\left(I_{\mathrm{KA}} / I_{\mathrm{Glu}}\right.$ of 0.82$)$ (Table 1).

Because I780L had significantly slowed desensitization, particularly in response to KA, we tested the triple mutant (525E/ 780L/784K), reproducing in GluR6 the immediate environment around R2 L483Y. The effect of the I780L mutation was additive, with desensitization slowed further compared with the K525E/ Q784K double mutant. The triple mutant desensitized nearly 11-fold slower than R6 WT in response to Glu and 29-fold slower than WT in response to KA (Fig. 3, Table 1). Prolonged agonist applications also revealed a significant change in the extent of desensitization (\%des), particularly for responses to KA. Steadystate currents were $\sim 13$ and $2 \%$ of peak for KA and Glu, respectively (Table 1). The high KA steady-state currents were not attributable to the action of KA as a partial agonist, however, because at this mutant KA was closer to being a full agonist $\left(I_{\mathrm{KA}} /\right.$ $I_{\mathrm{Glu}}$ of 0.85) (Table 1). Additional analysis showed that the increase in steady-state currents was attributable entirely to the mutation of I780 to leucine. The I780L mutant, in which $\tau_{\text {des }}$ was increased, had a large steady-state current in response to KA
(13\% of peak) and a clear, albeit much smaller, steady-state current evident in response to Glu (2\%) (Table 1). Again, as described above, for the single mutant, KA was closer to a full agonist, ruling out an effect of partial agonism. In contrast, for those I780 mutants in which $\tau_{\text {des }}$ was reduced (A, F, S, and V), desensitization was complete. Mutating I780 to leucine therefore has the dual effect of extending $\tau_{\text {des }}$ and reducing \%des, whereas other changes to this site have the opposite effects. If desensitization is modeled as a simple two state equilibrium, we would expect such a correlation between desensitization rate and extent (equivalent to the forward rate and equilibrium constants, respectively), other things being equal.

\section{Evidence for additional interactions across the dimer interface}

In combination, mutation of K525, I780, and Q784 to the residues found in GluR2 (E, L, and K, respectively) both slowed and reduced desensitization, but not to the extent observed in the R2 L483Y mutant. We therefore looked for additional residue differences between GluR2 L483Y and GluR6 that might account for their different desensitization phenotypes. Again using the GluR6 S1S2 domoate structure, we identified two additional residues that might be involved in intersubunit contacts around Y521. One of these, E787, may interact with Y521 directly, whereas the other, K696, may interact indirectly with Y521 via E787 (Fig. 4A) (supplemental Fig. 1, available at www.jneurosci.org as supplemental material). In the R6 S1S2 structure, the carboxyl side chain of E787 is located within $4 \AA$ of the main chain nitrogen of Y 521 , although the conformation of E787 is somewhat uncertain because of poor electron density (supplemental Fig. 1, available at www.jneurosci. org as supplemental material). We therefore do not know whether E787 interacts with Y521, but an equivalent hydrogen bond is evident in GluR2 dimers [R2 S1S2-AMPA (Armstrong and Gouaux, 2000)]. This site is highly conserved and is a glutamate in all non-NMDA receptor subunits. It is also very sensitive to mutagenesis. Mutation of the equivalent residue in the Xenopus laevis subunit XenU1 to lysine abolished kainate binding (Green et al., 2002). In GluR2, mutating the homologous site (E755) to glutamine accelerated desensitization 18 -fold, whereas the alanine mutant was nonfunctional (Horning and Mayer, 2004). Perhaps unsurprisingly, we found that mutation of E787 similarly affected GluR6 desensitization. R6 E787A responded to both Glu and KA with very small and rapidly desensitizing currents. Responses desensitized with time constants $\sim 1 \mathrm{~ms}$, and peak sizes were $1-2 \%$ of WT (Fig. $4 B$, Table 2). Responses recorded from R6 E787G were similar, with submillisecond $\tau_{\text {des }}$ and average peak currents $<100 \mathrm{pA}$ (Table 2). Even the conservative change E787D resulted in a receptor with significantly faster desensitization in response to both Glu and KA (1.3 \pm 0.1 ms for both) (Fig. 4B, Table 2). Current sizes for E787D were 
Table 2. Analysis of GluR6 dimer interface mutants

\begin{tabular}{|c|c|c|c|c|c|c|c|c|}
\hline \multirow[b]{2}{*}{ Mutant } & \multicolumn{4}{|l|}{ Glutamate } & \multicolumn{4}{|l|}{ Kainate } \\
\hline & $I_{\text {peak }}(\mathrm{nA})$ & $\tau_{\text {des }}(\mathrm{ms})$ & \%des & $n$ & $I_{\text {peak }}(n A)$ & $\tau_{\text {des }}(\mathrm{ms})$ & \%des & $n$ \\
\hline Wild type & $6.4 \pm 0.3$ & $5.7 \pm 0.1$ & $99.7 \pm 0.04$ & 80 & $4.6 \pm 0.3$ & $4.6 \pm 0.1$ & $99.5 \pm 0.1$ & 47 \\
\hline Y521A & $8.4 \pm 0.5$ & $31.3 \pm 3.1$ & $99.7 \pm 0.1$ & 11 & $7.8 \pm 0.6$ & $12.5 \pm 1.4$ & $99.8 \pm 0.1$ & 10 \\
\hline Y521H & $5.9 \pm 0.9$ & $18.5 \pm 2.8$ & $99.7 \pm 0.2$ & 5 & $4.9 \pm 1.0$ & $14.4 \pm 2.6$ & $98.4 \pm 0.5$ & 5 \\
\hline Y521L & $6.7 \pm 0.9$ & $19.6 \pm 1.1$ & $99.9 \pm 0.1$ & 13 & $5.9 \pm 0.8$ & $11.4 \pm 1.2$ & $99.9 \pm 0.1$ & 11 \\
\hline K696A & $8.3 \pm 0.8$ & $11.4 \pm 1.1$ & $99.4 \pm 0.5$ & 13 & $6.1 \pm 0.8$ & $6.9 \pm 0.6$ & $98.9 \pm 0.9$ & 12 \\
\hline K696E & $4.3 \pm 0.8$ & $11.9 \pm 1.5$ & 100 & 4 & $2.8 \pm 0.7$ & $3.6 \pm 0.1$ & 100 & 3 \\
\hline K696R & $7.6 \pm 1.1$ & $25.8 \pm 2.7$ & $98.6 \pm 0.8$ & 13 & $5.3 \pm 0.6$ & $15.5 \pm 1.8$ & $98.9 \pm 0.4$ & 11 \\
\hline E787A & $0.09 \pm 0.03$ & $0.8 \pm 0.1$ & 100 & 17 & $0.07 \pm 0.2$ & $1.2 \pm 0.1$ & 100 & 5 \\
\hline E787D & $1.9 \pm 0.3$ & $1.3 \pm 0.1$ & $99.9 \pm 0.1$ & 13 & $0.2 \pm 0.04$ & $1.3 \pm 0.1$ & $99.3 \pm 0.7$ & 10 \\
\hline $\mathrm{E} 787 \mathrm{G}$ & $0.06 \pm 0.02$ & $0.9 \pm 0.6$ & 100 & 8 & $0.08 \pm 0.03$ & $1.2 \pm 0.1$ & 100 & 5 \\
\hline Y521A & $3.8 \pm 0.8$ & $4.6 \pm 0.2$ & 100 & 10 & $2.7 \pm 0.7$ & $3.6 \pm 0.1$ & 100 & 10 \\
\hline \multicolumn{9}{|l|}{ I780L } \\
\hline Y521L & $0.9 \pm 0.3$ & $1.8 \pm 0.1$ & 100 & 12 & $0.2 \pm 0.1$ & $1.4 \pm 0.1$ & 100 & 11 \\
\hline \multicolumn{9}{|l|}{$\mathrm{I} 780 \mathrm{~A}$} \\
\hline Y521L & $8.3 \pm 0.9$ & $46.6 \pm 5.1$ & $99.3 \pm 0.2$ & 7 & $8.1 \pm 0.8$ & $38.5 \pm 5.5$ & $96.9 \pm 1.3$ & 8 \\
\hline \multicolumn{9}{|l|}{ I780L } \\
\hline K696R & $8.2 \pm 0.8$ & $175 \pm 16$ & $92.9 \pm 1.2$ & 9 & $8.1 \pm 0.8$ & $109 \pm 15$ & $86.0 \pm 2.4$ & 8 \\
\hline \multicolumn{9}{|l|}{ I780L } \\
\hline Y521L & $4.5 \pm 0.8$ & $28.1 \pm 3.1$ & $99.5 \pm 0.04$ & 7 & $4.2 \pm 0.8$ & $29.1 \pm 3.3$ & $97.3 \pm 0.6$ & 7 \\
\hline \multicolumn{9}{|l|}{ K525E } \\
\hline \multicolumn{9}{|l|}{ I780L } \\
\hline \multicolumn{9}{|l|}{ Q784K } \\
\hline K525E & $6.7 \pm 1.0$ & $346 \pm 55$ & $94.1 \pm 1.8$ & 11 & $5.9 \pm 0.9$ & $359 \pm 62$ & $67.6 \pm 4.5$ & 10 \\
\hline \multicolumn{9}{|l|}{ K696R } \\
\hline \multicolumn{9}{|l|}{ I780L } \\
\hline \multicolumn{9}{|l|}{ Q784K } \\
\hline Y521L & $7.4 \pm 1.6$ & $73.4 \pm 8.5$ & $98.1 \pm 1.1$ & 6 & $6.8 \pm 1.6$ & $56.8 \pm 3.0$ & $91.3 \pm 2.3$ & 10 \\
\hline \multicolumn{9}{|l|}{ K525E } \\
\hline \multicolumn{9}{|l|}{ K696R } \\
\hline \multicolumn{9}{|l|}{ I780L } \\
\hline Q784K & & & & & & & & \\
\hline
\end{tabular}

Categories are as described in Table 1, as is highlighting of values. The mutant K525E/K696R/1780L/0784K is referred to as the quadruple mutant in text.

larger in response to Glu (30\% of WT), but again responses to KA were small ( $4 \%$ of WT).

Unlike other mutants we generated with significantly attenuated currents in response to either Glu or KA, E787A and E787G had extremely small currents in response to both agonists. Desensitization, although fast, was not sufficient to explain the reduced peak responses, because E787D desensitized equally quickly but with more robust currents in response to Glu (Fig. $4 B$ ). We therefore investigated the expression of these mutants, using immunoblots to measure total and surface expression levels. Surface expression was determined after treatment of cells with a biotinylation reagent, solubilization of constituent proteins, and separation of biotinylated proteins on streptavidin beads (see Materials and Methods). Total expression of GluR6 did not vary greatly between WT and the E787A, D, or G site mutants (Fig. 4C). Surface expression, however, was reduced for all three mutants, but especially for E787G, in which no surfacelabeled GluR6 was evident, despite total expression levels that were similar to WT (Fig. 4C). This contrasted with the normal surface expression of I780A, another mutant with reduced current sizes (Fig. 4C). The small currents observed for E787G, and to a lesser extent E787A, therefore appear to be the result of reduced surface expression, presumably because of disrupted assembly and/or trafficking.

The second residue we identified from the GluR6 structure, K696, is within $\sim 3.5 \AA$ of E787 on the opposing subunit (Fig. $4 A$ ). Again, we cannot tell whether K696 and E787 interact (for example in a salt bridge) because of weak electron density for these two residues (supplemental Fig. 1, available at www. jneurosci.org as supplemental material). Such an interaction is certainly possible, with substitution of common lysine and glutamate side-chain rotamers enabling formation of a $2.4 \AA$ hydrogen bond between the N- and C-terminal groups of K696 and E787. Crucially for our study, the homologs of K696 are lysines in the kainate subunits GluR5 and GluR7 but arginines in AMPA subunits (Fig. 1). As such, it represents an additional point of difference between GluR6 and GluR2, with the potential to influence R6 Y521 (R2 L483) via R6 E787 (R2 E755). We therefore investigated the responses of R6 K696R to see whether this difference further defined the desensitization kinetics of GluR6 compared with GluR2 L483Y. This was indeed the case, with desensitization time constants significantly slower than WT for responses to both Glu (4.5-fold) and KA (3.4-fold) (Fig. 4D, Table 2). There were also small but significant steady-state currents with both agonists.

Modeling using the GluR6 S1S2 crystal structure showed that an arginine at position 696 could also potentially form single hydrogen bonds with E787 (ranging from 2.2 to $3.1 \AA$ between the glutamate carboxyl and arginine amine groups of common glutamate and arginine rotamers). Perhaps surprisingly, desensitization in response to Glu was also slowed when lysine was substituted to either alanine (K696A) or glutamate (K696E) (Table 2). GluR6 desensitization was therefore slowed by mutation of K696 to residues of opposing charges and significantly different sizes. This appears to rule out specific stabilization of the dimer by the mutant side chains, particularly because there was no obvious difference in the strength of potential interactions between E787 and either a lysine or arginine at position 696. It therefore appears that lysine itself is specifically required for fast and complete desensitization in GluR6. In other words, K696 must be 
involved in selective contacts that reduce the energy barrier to desensitization and/or increase the relative stability of the desensitized state. For example, lysine may destabilize the active state by steric or charge effects (unlikely if the GluR6 S1S2 domoate structure represents the active state conformation) or stabilize the desensitized state (or the transition state leading to it), for example via $\mathrm{H}$-bonds or a salt bridge.

We therefore identified four residues surrounding Y521 that differ between GluR6 and GluR2 and are involved in controlling desensitization rates. Comparing the effects of the component mutations (K525E/Q784K, K696R, and I780L) alone and in combination, they appeared to be additive, with successive mutations slowing desensitization (Tables 1,2). We therefore introduced all four changes into a mutant receptor. This quadruple mutant (R6 K525E/K696R/I780L/Q784K [R7(quad)]) desensitized 61-fold slower in response to Glu and 78-fold slower in response to KA than did WT receptors. In both cases, the time constants were more than one-third of a second (Fig. 4E, Table 2). There were also large steady-state currents for both Glu and KA responses ( $\sim 6$ and $32 \%$ of peak currents, respectively) (Fig. $4 E$, Table 2 ). By changing four sites, bringing the GluR6 primary sequence closer to that of GluR2 L483Y, we had markedly slowed the rate of desensitization, although with only limited effect on the extent of desensitization.

\section{Analysis of mutations to Y521}

These four residues represent all of the amino acid differences between GluR6 and GluR2 within $7 \AA$ of the key Y521/L483 site. We had already confirmed that the effect of the K525E/Q784K mutation was dependent on the presence of a tyrosine at position 521 (Fig. $2 B$ ), so we tested whether this was also true for the effects of mutations to K696 and I780. Incorporating Y521L into R6 K525E/K696R/I780L/Q784K [the R6(quad)], however, only partly reversed the effects of these mutations. Desensitization rates were still 12.9-fold and 12.3-fold slower than WT in response to Glu and KA, respectively (Table 2). This was also the case when Y521L was incorporated into R6 K525E/I780L/Q784K (the triple mutant), with desensitization rates that were 4.9 -fold and 6.3-fold slower than WT in response to Glu and KA (Table 2). Addition of Y521L to these mutants therefore accelerated desensitization by between twofold and sixfold but clearly did not revert their phenotype to WT. To investigate this further, we tested the effect of single mutations to R6 Y521. When changed to leucine, histidine $(\mathrm{H})$, or alanine, the rate of desensitization actually slowed by approximately threefold compared with WT (Fig. 5A, Table 2). The effect of the Y521L single mutant might seem counterintuitive, but direct comparison with GluR2/R3 is necessarily complicated by other residue differences. It is, for example, possible that the loss of the putative cation- $\pi$ interaction between Y521 and K525 allows closer contacts between subunits. A similar packing effect is possible for both $\mathrm{Y} 521 \mathrm{H}$ and Y521A, and in fact the equivalent mutation in GluR2 (L483A) also slows desensitization, the phenotype explained on the basis of steric effects (Horning and Mayer, 2004).

\section{Determining the nature of interactions between residues 521 and 780}

To investigate why changing Y521 to leucine in GluR6 did not completely reverse the effects of the multiple (triple and quadruple) mutants, we made a series of double mutants with I780. Because these residues are located next to each other across the dimer interface, interactions between the two may significantly affect mutant phenotypes. Single mutations to leucines at both

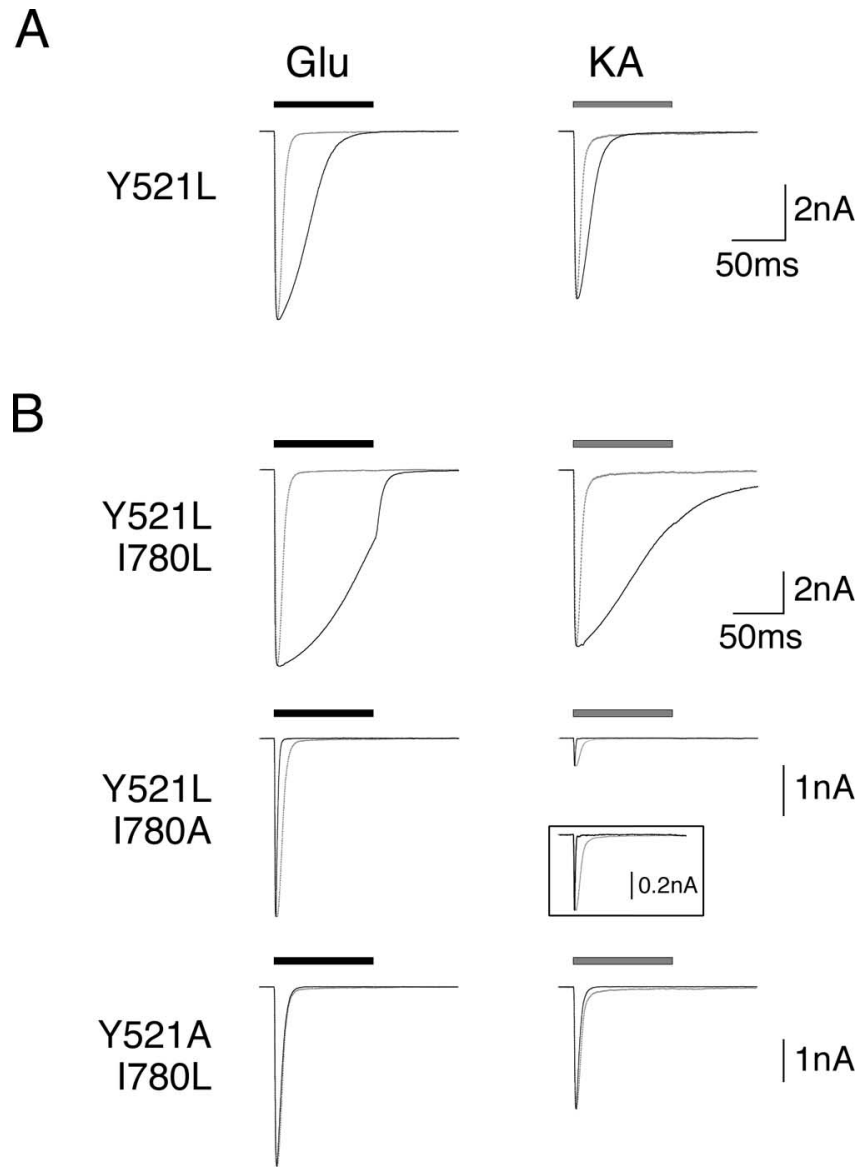

Figure 5. Investigation of interactions between residues 521 and 780 . $\boldsymbol{A}$, Responses to Glu ( 3 mm; black bars) and KA (1 mm; gray bars) recorded from HEK 293 cells expressing R6 Y521L. GluR6 WT traces (gray dotted) are overlaid and normalized to the mutant peak response. $\boldsymbol{B}$, Representative responses recorded from R6 Y521L/I780L, Y521L/I780A, and Y521A/I780L, shown as in $\boldsymbol{A}$. For responses showing slow initial desensitization, $\tau_{\text {des }}$ values were determined from fits to the portion of the response showing first-order kinetics (typically from 75 to $90 \%$ of peak).

sites slowed desensitization, whereas mutation to alanine had differing effects at the two sites (Figs. 3A, 5A). Desensitization of Y521A was also slowed (Table 2), whereas I780A accelerated the rate of desensitization, as well as greatly attenuated peak currents (Table 1). We therefore tested leucine and alanine mutants to Y521 and I780 in combination. The double leucine mutant (R6 Y521L/I780L) significantly slowed desensitization, by 8.2 -fold and 8.4-fold in response to Glu and KA, respectively (Fig. $5 B$, Table 2), as expected from two single mutants, which both slowed desensitization. It should be noted that this mutant, along with some others (i.e., Y521A and Y521L), exhibited slow initial desensitization. These were fitted as described in Materials and Methods to ensure that $\tau_{\text {des }}$ values were obtained from the region of the response showing first-order kinetics. We next tested the Y521L/I780A double mutant. This had very fast desensitization kinetics (Fig. 5B, Table 2), suggesting that the I780A phenotype was dominant. The reverse mutation (Y521A/I780L), in contrast, had a very different effect. Despite both single mutations slowing desensitization to a similar extent, the double mutant had responses very close to GluR6 WT (Fig. 5B, Table 2). This strongly indicates there is an interaction between the two residues (see Discussion). 
A

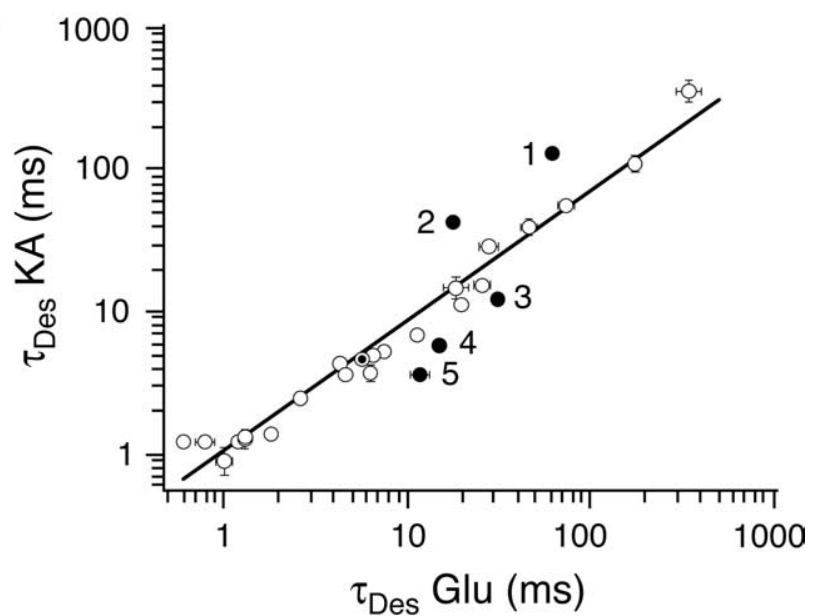

B

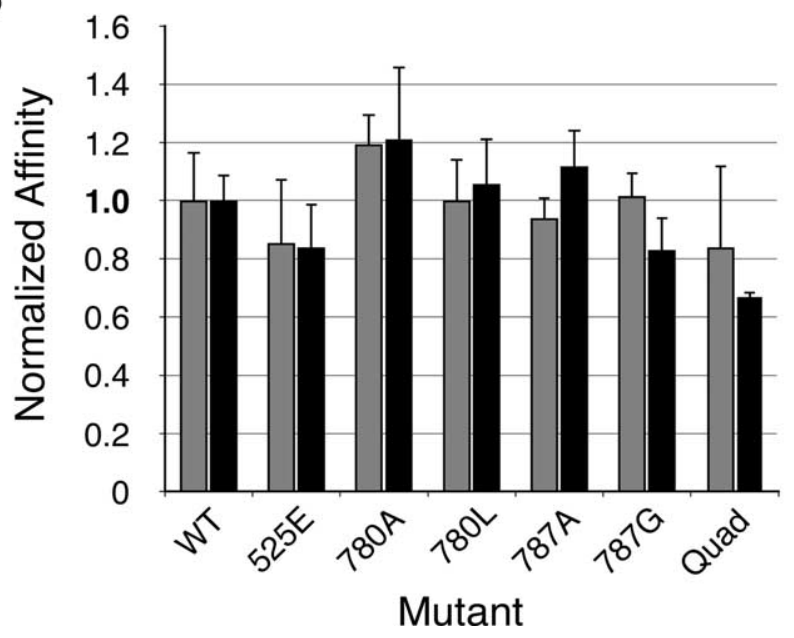

Figure 6. Desensitization and ligand-binding properties of GluR6 mutants. $A$, Desensitization time constants $\left(\tau_{\text {des }}\right)$ for KA plotted against $\tau_{\text {des }}$ for Glu, displayed on log scales for clarity. A line of best fit for all points is shown $\left(r^{2}=0.921\right)$. WT is indicated with a center dot. Outliers (in which the ratio of $\tau_{\text {des }}$ for Glu and KA differed by $>2$-fold compared with WT) are indicated by filled circles. These are as follows: K525E/1780L/0784K (1) and I780L (2), both having a larger effect on KA desensitization; and Y521A (3), K525E/Q784K (4), and K696E (5), with a larger effect on Glu desensitization. $B$, Bar graph showing ligand-binding affinities for KA (gray bars; $K_{\mathrm{d}}$ values) and Glu (black bars; $K_{\mathrm{i}}$ values), determined as described in Materials and Methods and normalized to WT. Error bars show normalized SEMs (Table 3).

\section{Desensitization rates, extent, and binding affinities}

In their study of GluR6 dimer interface mutants, Fleck et al. (2003) observed a correlation between Glu and KA desensitization time constants ranging between 1 and $5 \mathrm{~ms}$. Our data showed a similar correlation in $\tau_{\mathrm{des}}$ but extending over almost three orders of magnitude (Fig. 6A). For all of the mutants described here, the correlation coefficient $\left(r^{2}\right)$ was 0.921 , but there were a number of distinct outliers (in which the ratio of Glu and KA desensitization rates differed by more than twofold compared with WT). When these were excluded, the correlation was even stronger $\left(r^{2}=0.966\right)$. The outliers fell into two groups: one in which KA desensitization was slowed to a greater extent than Glu desensitization (Fig. $6 A$, points 1,2 ), and one in which Glu rates slowed disproportionately (Fig. 6A, points $3-5$ ). We looked for additional correlations between the desensitization rate and other kinetic properties. There was no strong linear relationship between $\tau_{\text {des }}$ and steady-state currents, although the two were weakly correlated (e.g., $r^{2}=0.71$ for Glu), consistent with the
Table 3. Ligand affinities of selected mutants

\begin{tabular}{lcclll}
\hline & \multicolumn{1}{l}{ Kainate } & & & \multicolumn{2}{l}{ Glutamate } \\
\cline { 2 - 3 } Mutant & $K_{\mathrm{d}}(\mathrm{nM})$ & & & $K_{\mathrm{i}}(\mathrm{nm})$ & $n$ \\
\hline Wild type & $8.9 \pm 1.5$ & 3 & $320 \pm 29$ & 3 \\
K525E & $7.6 \pm 2.0$ & 4 & $266 \pm 50$ & 3 \\
1780A & $10.6 \pm 1.0$ & 3 & $387 \pm 81$ & 4 \\
1780L & $8.9 \pm 1.3$ & 3 & $337 \pm 50$ & 3 \\
E787A & $8.3 \pm 0.7$ & 4 & $357 \pm 41$ & 3 \\
E787G & $9.0 \pm 0.8$ & 5 & $265 \pm 37$ & 3 \\
K525E/K696R/ & $7.4 \pm 2.6$ & 3 & $214 \pm 7$ & 3 \\
1780L/Q784K & & & & \\
\hline
\end{tabular}

$K_{\mathrm{d}}$ is the affinity constant determined for $\left[{ }^{3} \mathrm{H}\right]$ kainate, and $K_{\mathrm{i}}$ is the estimated affinity constant, both shown as mean \pm SEM for $n$ values, determined as described in Materials and Methods.

link between rate and equilibrium for a two-state process. We found no correlation between $\tau_{\text {des }}$ and the rate of deactivation (supplemental Table 1, available at www.jneurosci.org as supplemental material) for either Glu or KA responses or between large steady-state currents and reduced agonist efficacy (measured as $\left.I_{\mathrm{KA}} / I_{\mathrm{Glu}}\right)$. In fact, all mutants with significant steady-state currents showed higher KA efficacy than R6 WT (Tables 1, 2), although there was no linear correlation. We did, however, find some agonist-specific effects on the size of steady-state currents for mutants containing either the I780L or K696R mutations. Receptors carrying the I780L mutation (with the notable exception of Y521A/I780L) had steady-state currents $>2.5 \%$ in response to KA (Tables 1,2) but smaller steady-states in response to Glu. For mutants containing arginine at 696, \%des in response to Glu was disproportionately affected, with all K696R mutants having steady-state currents $>1.4 \%$ (Tables 1,2 ).

In the light of these agonist-specific differences in desensitization phenotypes, we also looked for changes in binding affinities, testing a selection of mutants with significantly altered desensitization rates and/or extents. Membranes were isolated from HEK 293 cells expressing R6 WT and mutant subunits. Kainate affinity $\left(K_{\mathrm{d}}\right)$ was determined by saturation binding with tritiated kainate, whereas glutamate affinity $\left(K_{\mathrm{i}}\right)$ was estimated from $\mathrm{IC}_{50}$ values determined in displacement assays. We observed no significant change in the affinity of either kainate or glutamate for any of the mutants tested (Fig. 6B, Table 3). This was the case both for mutations that significantly accelerated (e.g., E787A) or slowed (e.g., K525E/K696R/I780L/Q784K) desensitization and for mutations with significantly increased steady-state responses (e.g., I780L). From these data, it can be inferred that our mutations have not significantly affected the ligand binding pocket and are instead influencing desensitization through changes within the dimer interface.

\section{Discussion}

\section{Mutant phenotypes and the dimer stabilization model}

One aim of this study was to determine why GluR6 desensitizes quickly and completely, although it has a tyrosine (Y521) at the homologous site to the nondesensitizing R2 L483Y mutant. The cumulative effect of changing four residues neighboring R6 Y521 that differ in GluR2 was much greater on the rate than on the extent of desensitization. Our GluR6(quad) mutant desensitized slowly but almost completely ( $\tau_{\text {des }}$ of $\sim 350 \mathrm{~ms}$; \%des of $94 \%$ for Glu, 67\% for KA). In contrast, the nondesensitizing R2 L483Y mutant desensitizes slowly and only minimally in response to glutamate ( $\tau_{\text {des }}$ of $164 \mathrm{~ms}$; \%des of $8 \%$ ) (Sun et al., 2002). The quadruple mutation therefore has a greater apparent effect on the energy barrier to desensitization (rate) than on the relative stability of the open and desensitized states (extent). The difference 
between the GluR6(quad) and GluR2 L483Y mutants could be attributable to either a more stable desensitized state in GluR6 or a relatively weaker dimer association.

Both appear to play a role, although quantification would require direct measurement of dimer stability. GluR6 WT desensitizes more completely than GluR2, indicating a more stable desensitized state. This may be related to the higher ligand affinities of kainate subunits compared with their AMPA counterparts and the more extensive binding-cleft interactions observed in high-resolution GluR5 and GluR6 structures (Mayer, 2005). Higher-affinity binding could tend to drive the receptor complex into the desensitized state, making it energetically "harder" to completely block desensitization. Greater WT desensitization is reflected in estimates of $\Delta G_{\text {des }}$ (see Materials and Methods). Compared with WT, GluR6(quad) stabilizes the open state relative to the desensitized state by $\sim 1.8 \mathrm{kcal} / \mathrm{mol}$ for Glu $(\sim 2.7$ $\mathrm{kcal} / \mathrm{mol}$ for KA). This is similar to the $\sim 2.6 \mathrm{kcal} / \mathrm{mol}$ stabilization for R2 L483F, although still much less than the $\sim 4.3 \mathrm{kcal} /$ mol stabilization for R2 L483Y (Sun et al., 2002). Were the GluR6(quad) dimer to be stabilized to the same extent as R2 L483Y, it would desensitize by $\sim 20 \%$ ( 12\% for KA). Lower overall dimer stability in GluR6 must account for this remaining discrepancy. One factor may be the different points of contact observed in the GluR2 and GluR6 dimer interfaces (Armstrong et al., 1998; Nanao et al., 2005), which may limit the capacity of our mutations to stabilize the dimer. These interface differences are unlikely to result from the partially closed conformation of the GluR6-domoate complex, because the dimer interface in GluR2 is unaffected by domain closure angle (Armstrong and Gouaux, 2000).

Another aim of this work was to determine whether desensitization in kainate receptors involves dimer rearrangement, as found in AMPA receptors. Overall, our mutant phenotypes did support a dimer-rearrangement model for GluR6. For the K525E and Q784K mutants, only the double mutant slowed desensitization, consistent with a switch from an intrasubunit to an intersubunit cation- $\pi$ interaction stabilizing the dimer (Fig. $2 \mathrm{~B}$, Table 1). Desensitization rates reverted to normal in Y521L/K525E/ Q784K (Fig. 2B), demonstrating the importance of the Y521 $\pi$ electrons. Published mutagenesis studies support this interpretation. In GluR3, mutating the homolog of R6 K525 (E511) to lysine has no effect on its own but partially reverses the desensitization block in R3 L507Y (Stern-Bach et al., 1998), presumably as a result of the formation of a disruptive interaction between E511K and the tyrosine. Our data for R6 Q784K agrees with published findings (Fleck et al., 2003), whereas mutating the GluR2 homolog of this site (K752) to an alanine in the L483Y mutant increases both the rate and extent of desensitization (Sun et al., 2002). Single mutants (R2 K752A or M) slow desensitization, but, with a leucine at position 483 in WT GluR2, these changes may strengthen dimer contacts (Horning and Mayer, 2004).

The effects of R6 I780 mutations also fit with the "dimerrearrangement" model (Table 1). In WT GluR6, isoleucine does not pack particularly closely with the neighboring Y521 (Fig. 2 A). Slower desensitization of $1780 \mathrm{~L}$ is consistent with leucine stabilizing the dimer through tighter packing, whereas faster desensitization of Ala, Ser, Val, and Phe mutations can be explained by poorer dimer packing (and therefore stability). Mutations to K696 were perhaps the most interesting in terms of providing clues as to how GluR6 subunits move during receptor gating. Introducing arginine, glutamate, and alanine all slowed desensitization, respectively, extending, reversing the charge, and trun- cating the side chain. Specific contacts in these mutants cannot account for their common phenotype, so it must be lysine that makes the key contacts, promoting desensitization by either destabilizing the active state or stabilizing the desensitized state (or an associated transition state). Overall, we identified four residue differences that help explain why GluR6 desensitizes whereas the R2 L483Y mutant does not, although the goal of generating a nondesensitizing kainate receptor remains elusive.

\section{The role of residue 521 in GluR6 desensitization}

Although most of our mutations fitted the dimer-rearrangement model of desensitization, changes to Y521 had some unpredicted results. For example, the effects of mutations to K696 and I780 were not dependent on the tyrosine at position 521. Combination of the triple and quadruple mutants with Y521L only partially reverted their phenotypes to WT, whereas both Y521L and Y521L/I780L unexpectedly desensitized more slowly than WT GluR6 (Table 2). We hypothesize that the Y521L mutation results in closer packing after the loss of the putative Y521-K525 interaction and that dimer packing is further improved in the Y521L/ I780L double mutant. This would explain the partial effect of Y521L when incorporated into the triple and quadruple mutants. It also illustrates an important point. Although the side chain at position 521 in GluR6 strongly influences desensitization properties, it does not act as a desensitization switch in the same way as its homolog in AMPA receptors. Indeed, the difficulty in recreating this all-or-nothing effect in a kainate subunit highlights the serendipitous nature of the original R3 L507Y nondesensitizing mutant.

Investigating the interplay between Y521 and I780 further, we found that Y521L/I780L desensitized slowly, Y521L/I780A desensitized rapidly, and Y521A/I780L responses were like WT (Fig. $5 B)$. This last mutant was particularly interesting, because its component single mutants (Y521A and I780L) both slowed desensitization. With these two mutants increasing the relative stability of the open state on their own, but not when combined, their phenotypes must be the result of an interaction between the two residues. This is not unexpected given their close proximity in the structure but again underlines the complex nature of the interactions controlling desensitization kinetics in GluR6. With leucines replacing both Y521 and I780, the primary sequence of GluR6 is closer to that of GluR2, but desensitization is slowed. Only by reverting 521 to tyrosine, however, was it possible to slow desensitization further, as observed in the triple and quadruple mutants.

\section{Desensitization and conformational change}

We found that desensitization rates for Glu and KA were for the most part closely correlated (Fig. 6A). There was more variation in steady-state current sizes, with changes to residues K696 and I780 having a disproportionate effect on \%des in response to Glu and KA, respectively. This does not appear to be attributable to changes in agonist efficacy (e.g., from a full to a partial agonist), because $I_{\mathrm{KA}} / I_{\mathrm{Glu}}$ was not correlated with either the relative desensitization rates or steady-state current sizes, nor is it attributable to changes in the binding pocket, because the affinity constants for Glu or KA were unaffected (Table 3). We conclude instead that the overall conformational changes accompanying desensitization induced by Glu and KA must differ, such that residue 696 has more influence on the former, and residue 780 more influence on the latter.

The ultimate goal of studies of this nature is to define the conformation of iGluRs in the resting, open, and desensitized 
states. Structural studies have helped determine movements in the S1S2 domain accompanying ligand binding in both AMPA and kainate receptors. There is also good evidence, particularly from studies of partial agonists, that the GluR2 S1S2 dimer conformation equates to the active receptor (Armstrong et al., 2003; Jin et al., 2003). Although gating mechanisms appear to differ between AMPA and kainate receptors (Bowie, 2002; Bowie and Lange, 2002), our data are consistent with the GluR6 dimer also equating to an active conformation. This supports a common model for non-NMDA receptor desensitization, in which the key step is the rearrangement of the dimer interface.

Less is known about the conformation of the desensitized state. Kinetic modeling has revealed some of its properties in AMPA receptors (Robert and Howe, 2003), and concanavalin-A has been used to study desensitization in kainate receptors (Fay and Bowie, 2006). Although many questions remain, these studies are consistent with a model in which the agonist binding domain moves as a rigid body, with the S1S2 dimer interaction either being rearranged or lost. In this context, residues K696 and E787 are interesting, because their phenotypes are poorly explained by reference to the current GluR6 structure. We would therefore speculate that these residues are involved in interactions in the desensitized state (or transition to it). Future identification of interactions with these sites may therefore prove important for defining the desensitized conformation of the S1S2 domain in kainate receptors.

\section{References}

Armstrong N, Gouaux E (2000) Mechanisms for activation and antagonism of an AMPA-sensitive glutamate receptor: crystal structures of the GluR2 ligand binding core. Neuron 28:165-181.

Armstrong N, Sun Y, Chen G-Q, Gouaux E (1998) Structure of a glutamatereceptor ligand-binding core in complex with kainate. Nature 395:913-917.

Armstrong N, Mayer M, Gouaux E (2003) Tuning activation of the AMPAsensitive GluR2 ion channel by genetic adjustment of agonist-induced conformational changes. Proc Natl Acad Sci USA 100:5736-5741.

Bowie D (2002) External anions and cations distinguish between AMPA and kainate receptor gating mechanisms. J Physiol (Lond) 539:725-733.

Bowie D, Lange GD (2002) Functional stoichiometry of glutamate receptor desensitization. J Neurosci 22:3392-3403.

Fay AM, Bowie D (2006) Concanavalin-A reports agonist-induced conformational changes in the intact GluR6 kainate receptor. J Physiol (Lond) 572:201-213.

Fleck MW, Cornell E, Mah SJ (2003) Amino-acid residues involved in glu- tamate receptor 6 kainate receptor gating and desensitization. J Neurosci 23:1219-1227.

Furukawa H, Singh SK, Mancusso R, Gouaux E (2005) Subunit arrangement and function in NMDA receptors. Nature 438:185-192.

Green T, Rogers CA, Contractor A, Heinemann SF (2002) NMDA receptors formed by NR1 in Xenopus oocytes do not contain the endogenous subunit XenU1. Mol Pharmacol 61:326-333.

Horning MS, Mayer ML (2004) Regulation of AMPA receptor gating by ligand binding core dimers. Neuron 41:379-388.

Jin R, Banke TG, Mayer ML, Traynelis SF, Gouaux E (2003) Structural basis for partial agonist action at ionotropic glutamate receptors. Nat Neurosci 6:803-810.

Jin R, Clark S, Weeks AM, Dudman JT, Gouaux E, Partin KM (2005) Mechanism of positive allosteric modulators acting on AMPA receptors. J Neurosci 25:9027-9036.

Jones MV, Westbrook GL (1996) The impact of receptor desensitization on fast synaptic transmission. Trends Neurosci 19:96-101.

Lynch G (2002) Memory enhancement: the search for mechanism-based drugs. Nat Neurosci [Suppl] 5:1035-1038.

Mayer ML (2005) Crystal structures of the GluR5 and GluR6 ligand binding cores: molecular mechanisms underlying kainate receptor selectivity. Neuron 45:539-552.

Nanao MH, Green T, Stern-Bach Y, Heinemann SF, Choe S (2005) Structure of the kainate receptor subunit GluR6 agonist-binding domain complexed with domoic acid. Proc Natl Acad Sci USA 102:1708-1713.

Patneau DK, Mayer ML, Jane DE, Watkins JC (1992) Activation and desensitization of AMPA/kainate receptors by novel derivatives of willardiine. J Neurosci 12:595-606.

Robert A, Howe JR (2003) How AMPA receptor desensitization depends on receptor occupancy. J Neurosci 23:847-858.

Rosenmund C, Stern-Bach Y, Stevens CF (1998) The tetrameric structure of a glutamate receptor channel. Science 280:1596-1599.

Smith TC, Howe JR (2000) Concentration-dependent substate behavior of native AMPA receptors. Nat Neurosci 3:992-997.

Smith TC, Wang LY, Howe JR (2000) Heterogeneous conductance levels of native AMPA receptors. J Neurosci 20:2073-2085.

Stern-Bach Y, Russo S, Neuman M, Rosenmund C (1998) A point mutation in the glutamate binding site blocks desensitization of AMPA receptors. Neuron 21:907-918.

Sun Y, Olson R, Horning M, Armstrong N, Mayer M, Gouaux E (2002) Mechanism of glutamate receptor desensitization. Nature 417:245-253.

Swanson GT, Gereau IV RW, Green T, Heinemann SF (1997) Identification of amino acid residues that control functional behavior in GluR5 and GluR6 kainate receptors. Neuron 19:913-926.

Tichelaar W, Safferling M, Keinanen K, Stark H, Madden DR (2004) The three-dimensional structure of an ionotropic glutamate receptor reveals a dimer-of-dimers assembly. J Mol Biol 344:435-442.

Yelshansky MV, Sobolevsky AI, Jatzke C, Wollmuth LP (2004) Block of AMPA receptor desensitization by a point mutation outside the ligandbinding domain. J Neurosci 24:4728-4736. 